\title{
Dielectric spectroscopy of single cells: time domain analysis using Maxwell's mixture equation
}

\author{
Tao Sun, Shady Gawad, Nicolas G Green and Hywel Morgan ${ }^{1}$ \\ School of Electronics and Computer Science, University of Southampton, SO17 1BJ, UK
}

Received 12 July 2006, in final form 18 September 2006

Published 15 December 2006

Online at stacks.iop.org/JPhysD/40/1

\begin{abstract}
Dielectric spectroscopy is a powerful tool for investigating the dielectric properties of biological particles in suspension. For low volume fractions, the dielectric properties of the particles are related to the measured properties of the suspension by Maxwell's mixture equation. A number of different techniques can be used to measure the dielectric spectrum in the frequency domain or the time domain. In time domain dielectric spectroscopy, data can be converted into the frequency domain using convolution or Fourier transform, prior to data analysis. In this paper, we present a general method for transforming Maxwell's mixture equation from the frequency domain to the time domain allowing analysis of cell dielectric properties directly in the time domain. The derivation is based on the Laplace transform of the single shell model for a spherical particle, and can be extended to the multi-shell model. For a single shelled cell two characteristic relaxation time constants are derived. The results are compared with published analytical models. We show that the original frequency dependent mixture equation can be recovered by Fourier transform back to the frequency domain. As a result, a general relationship for the dielectric response of a mixture of particles is presented which links the frequency and time domains.
\end{abstract}

\section{Introduction}

The complex dielectric properties of suspensions of particles, such as cells, bacteria, water-oil emulsions and viruses have been studied extensively for many years. Measurements provide information about the structure, function and electrical characteristics of the system. The early work of Maxwell [1] and Wagner [2], Fricke [3-5], Cole [6-8] and Schwan [9-12], amongst others, laid the foundations for this field of research; see [13] for a review. The frequency dependent dielectric properties of a mixture of membrane-bound particles are characterized by a pronounced dielectric relaxation in the 1$100 \mathrm{MHz}$ range which is due to interfacial charging, and was termed the $\beta$-relaxation (for cells) by Schwan [10]. A cell has a thin insulating membrane, and the measured permittivity of a suspension of cells has a very high value at low frequencies due to charging of this membrane. As the frequency increases, the value of the permittivity decreases, approaching that of the suspending medium.

1 Author to whom any correspondence should be addressed.
The most widely used technique for analysis of cell suspensions is broad-band frequency domain dielectric spectroscopy. For suspensions of low volume fraction, the properties of the particles are related to the properties of the suspension through the well-known mixture theory, developed by Maxwell and Wagner 1. This approach works well for volume fractions less than $10 \%$ and the analysis was extended for higher concentrations by Bruggeman [14] and Hanai [15-17]. For the specific case of a particle with a thin single shell, i.e. a cell, suspended in a homogeneous suspending medium, there are two characteristic relaxation frequencies (or time constants in the time domain). This is because the poorly conducting membrane separates the cytoplasm and the suspending medium and it takes time to charge the membrane through the conducting phase in and outside the cell membranes [18]. The lower frequency dispersion $(<20 \mathrm{MHz})$ is related to the polarization of the cell membrane and measurement of this parameter provides information on the dielectric properties of the particle, particularly the capacitance of the membrane. The higher frequency dispersion 
$(>50 \mathrm{MHz})$ is the interfacial relaxation of the cell interior and exterior, as the membrane is effectively short-circuited at higher frequencies. The size of this dispersion is generally very small and not evident in measurements except in specific cases. For example, Zhang et al [19] measured the dielectric relaxation of polystyrene microcapsules and measured two relaxations, termed the $P$ and $Q$ dispersion.

Over the last decade, new high speed measurement techniques have been developed [20] that are based on time domain methods rather than frequency sweep methods. These approaches enable broad-band frequency spectra to be obtained in a short time period. Data is collected in the time domain and then converted into the frequency domain using methods such as convolution or Fourier transform. Data analysis is generally performed in the frequency domain. In certain cases it would be advantageous to be able to analyse and simulate the time-dependent behaviour of a collection of particles directly in the time domain. Examples include the behaviour of particles (cells) that move in the electric field or measurement of dynamic processes occurring in cells, for example chemical or electric field-induced changes (e.g. lysis or electroporation).

In this paper, we present a general method for the derivation of Maxwell's mixture equation in the time domain, based on the Laplace transformation. The approach is similar to that used by Kotnik et al [21] to analyse the transmembrane voltage of a cell during electroporation and electrofusion, and Foster [22] who used the Laplace transform to calculate the impulse response for the inner and outer membranes of a cell. In our work, the time constants for a single shelled cell model are analytically solved, taking into account the volume fraction. Finally, an analytical expression for the dielectric properties of a cell in suspension is derived in the time domain and compared with the well-known frequency domain expression.

\section{Theoretical calculations}

The theoretical derivation of the time domain expression of Maxwell's mixture formula is based on the following three assumptions.

A. The cell is assumed to be homogeneous and spherical and modelled using the single shell model.

B. The cell is located in a uniform electric field.

C. The medium is considered non-dispersive in the frequency range of interest.

For a spherical particle dispersed in a suspending medium at a low volume fraction, $\varphi(\ll 1)$, Maxwell's mixture equation (see [23] for the derivation) gives the steady-state value of the equivalent complex dielectric permittivity of the mixture in the complex frequency $(j \omega)$ according to

$$
\tilde{\varepsilon}_{\mathrm{mix}}=\tilde{\varepsilon}_{\mathrm{m}} \frac{1+2 \varphi \tilde{f}_{\mathrm{CM}}}{1-\varphi \tilde{f}_{\mathrm{CM}}},
$$

where $\tilde{\varepsilon}_{\text {mix }}, \tilde{\varepsilon}_{\mathrm{m}}, \tilde{\varepsilon}_{\mathrm{p}}$ are the complex permittivity for the mixture, suspending medium and the particle, respectively, and $\tilde{f}_{\mathrm{CM}}$ is the complex Clausius-Mossotti factor

$$
\tilde{f}_{\mathrm{CM}}=\frac{\tilde{\varepsilon}_{\mathrm{p}}-\tilde{\varepsilon}_{\mathrm{m}}}{\tilde{\varepsilon}_{\mathrm{p}}+2 \tilde{\varepsilon}_{\mathrm{m}}} .
$$

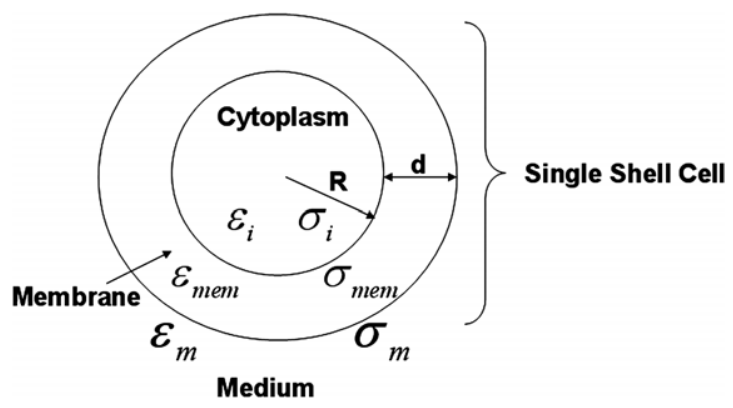

Figure 1. Schematic diagram of a single shelled spherical particle in a homogeneous suspending medium.

For a single shelled cell model, $\tilde{\varepsilon}_{\mathrm{p}}$ is given by

$$
\tilde{\varepsilon}_{\mathrm{p}}=\tilde{\varepsilon}_{\mathrm{mem}} \frac{\gamma^{3}+2 K_{23}}{\gamma^{3}-K_{23}},
$$

where

$$
K_{23}=\frac{\tilde{\varepsilon}_{i}-\tilde{\varepsilon}_{\mathrm{mem}}}{\tilde{\varepsilon}_{i}+2 \tilde{\varepsilon}_{\mathrm{mem}}} \quad \text { and } \quad \gamma=\frac{R+d}{R} .
$$

Here $\tilde{\varepsilon}_{\text {mem }}$ and $\tilde{\varepsilon}_{\mathrm{i}}$ are the complex permittivity of the cell membrane and cytoplasm, respectively. $R$ is the inner radius of the cell and $d(d \ll R)$ is the thickness of the membrane, as shown in figure 1. A general complex permittivity is given by

$$
\tilde{\varepsilon}(j \omega)=\varepsilon+\frac{\sigma}{j \omega},
$$

where $\varepsilon$ is the permittivity, $\sigma$ is the conductivity and $\omega$ is the angular frequency.

In order to link the frequency domain solution to the time domain, we replace $j \omega$ by the parameter $s$ in equation (4), leading to

$$
\tilde{\varepsilon}(s)=\varepsilon+\frac{\sigma}{s} .
$$

Using this parameter in equation (3) gives

$$
\begin{aligned}
\tilde{\varepsilon}_{\mathrm{p}}(s)= & \frac{\varepsilon_{\mathrm{mem}} s+\sigma_{\mathrm{mem}}}{s} \\
\times\{s & s\left[\gamma^{3}\left(\varepsilon_{\mathrm{i}}+2 \varepsilon_{\mathrm{mem}}\right)+2\left(\varepsilon_{\mathrm{i}}-\varepsilon_{\mathrm{mem}}\right)\right]+\gamma^{3}\left(\sigma_{\mathrm{i}}+2 \sigma_{\mathrm{mem}}\right) \\
& \left.+2\left(\sigma_{\mathrm{i}}-\sigma_{\mathrm{mem}}\right)\right\} \\
\times & s \\
& s\left[\gamma^{3}\left(\varepsilon_{\mathrm{i}}+2 \varepsilon_{\mathrm{mem}}\right)-\left(\varepsilon_{\mathrm{i}}-\varepsilon_{\mathrm{mem}}\right)\right]+\gamma^{3}\left(\sigma_{\mathrm{i}}+2 \sigma_{\mathrm{mem}}\right) \\
& \left.-2\left(\sigma_{\mathrm{i}}-\sigma_{\mathrm{mem}}\right)\right\}^{-1}
\end{aligned}
$$

Substituting equation (6) into equation (2) gives the ClausiusMossotti factor in terms of the parameter $s$ :

$$
\tilde{f}_{\mathrm{CM}}(s)=\frac{A\left(\varepsilon_{\mathrm{mem}} s+\sigma_{\mathrm{mem}}\right)-B\left(\varepsilon_{\mathrm{m}} s+\sigma_{\mathrm{m}}\right)}{A\left(\varepsilon_{\mathrm{mem}} s+\sigma_{\mathrm{mem}}\right)+2 B\left(\varepsilon_{\mathrm{m}} s+\sigma_{\mathrm{m}}\right)} .
$$

The expressions for the coefficients $A$ and $B$ are given in appendix I. Substituting equation (7) into equation (1) gives the complex permittivity of the mixture in the $s$-domain as

$$
\tilde{\varepsilon}_{\text {mix }}(s)=\frac{\left(\varepsilon_{\mathrm{m}} s+\sigma_{\mathrm{m}}\right)(C+2 \varphi D)}{s(C-\varphi D)} .
$$


Expanding the coefficients $C$ and $D$, (given in appendix I) then equation (8) is written as

$$
\begin{aligned}
\tilde{\varepsilon}_{\text {mix }}(s) & =\frac{\left(\varepsilon_{\mathrm{m}} s+\sigma_{\mathrm{m}}\right)\left(a_{1} s^{2}+a_{2} s+a_{3}\right)}{s\left(b_{1} s^{2}+b_{2} s+b_{3}\right)} \\
& =\frac{a_{1}\left(\varepsilon_{\mathrm{m}} s+\sigma_{\mathrm{m}}\right)\left(s-s_{1}\right)\left(s-s_{2}\right)}{b_{1} s\left(s-s_{3}\right)\left(s-s_{4}\right)} .
\end{aligned}
$$

The coefficients $a_{n}$ and $b_{n}(n=1,2,3)$ are given in appendix I. The zeros in the numerator and poles in the denominator $s_{k}$ $(k=1,2,3,4)$ are given by

$$
\begin{aligned}
s_{1,2} & =\frac{-a_{2} \pm \sqrt{a_{2}^{2}-4 a_{1} a_{3}}}{2 a_{1}}, \\
s_{3,4} & =\frac{-b_{2} \pm \sqrt{b_{2}^{2}-4 b_{1} b_{3}}}{2 b_{1}} .
\end{aligned}
$$

For the inverse Laplace transform, equation (9) is written as

$$
\begin{aligned}
\tilde{\varepsilon}_{\text {mix }}(s) & =\frac{\varepsilon_{\mathrm{m}} a_{1}}{b_{1}}+\frac{c_{1} s^{2}+c_{2} s+c_{3}}{b_{1} s^{3}+b_{2} s^{2}+b_{3} s} \\
& =\frac{\varepsilon_{\mathrm{m}} a_{1}}{b_{1}}+\frac{c_{1}\left(s-s_{5}\right)\left(s-s_{6}\right)}{b_{1} s\left(s-s_{3}\right)\left(s-s_{4}\right)},
\end{aligned}
$$

where the coefficients $c_{n}(n=1,2,3)$ are given by

$$
\begin{aligned}
& c_{1}=\varepsilon_{\mathrm{m}} a_{2}+\sigma_{\mathrm{m}} a_{1}-\frac{\varepsilon_{\mathrm{m}} a_{1} b_{2}}{b_{1}}, \\
& c_{2}=\varepsilon_{\mathrm{m}} a_{3}+\sigma_{\mathrm{m}} a_{2}-\frac{\varepsilon_{\mathrm{m}} a_{1} b_{3}}{b_{1}}, \\
& c_{3}=\sigma_{\mathrm{m}} a_{3}
\end{aligned}
$$

and the zeros $s_{5}$ and $s_{6}$ are given by

$$
s_{5,6}=\frac{-c_{2} \pm \sqrt{c_{2}^{2}-4 c_{1} c_{3}}}{2 c_{1}} .
$$

Applying the inverse Laplace transform to equation (11) gives the time domain expression for the mixture equation

$$
\varepsilon_{\text {mix }}(t)=\frac{\varepsilon_{\mathrm{m}} a_{1}}{b_{1}} \delta(t)+\left(k_{1}+k_{2} \mathrm{e}^{-t / \tau_{1}}+k_{3} \mathrm{e}^{-t / \tau_{2}}\right) U_{0}(t),
$$

where $\delta(t)$ is the unit impulse function, $U_{0}(t)$ is the unit step function and the coefficients $k_{n}(n=1,2,3)$ are given by

$$
\begin{aligned}
& k_{1}=\frac{c_{1} s_{5} s_{6}}{b_{1} s_{3} s_{4}}=\frac{\sigma_{\mathrm{m}} a_{3}}{b_{3}}, \\
& k_{2}=\frac{c_{1}\left(s_{3}-s_{5}\right)\left(s_{3}-s_{6}\right)}{b_{1} s_{3}\left(s_{3}-s_{4}\right)}, \\
& k_{3}=\frac{c_{1}\left(s_{4}-s_{5}\right)\left(s_{4}-s_{6}\right)}{b_{1} s_{4}\left(s_{4}-s_{3}\right)} .
\end{aligned}
$$

The two time constants $\tau_{1}$ and $\tau_{2}$ in equation (14) are given by

$$
\begin{gathered}
\tau_{1}=-\frac{1}{s_{3}}=\frac{2 b_{1}}{b_{2}-\sqrt{b_{2}^{2}-4 b_{1} b_{3}}}, \\
\tau_{2}=-\frac{1}{s_{4}}=\frac{2 b_{1}}{b_{2}+\sqrt{b_{2}^{2}-4 b_{1} b_{3}}} .
\end{gathered}
$$

The complex permittivity and conductivity are related by

$$
\tilde{\sigma}_{\text {mix }}(s)=s \tilde{\varepsilon}_{\text {mix }}(s) .
$$

The time domain expression for the conductivity can be derived by a similar procedure, as shown in appendix I.

\section{Discussion}

\subsection{Characteristic relaxation time constant}

As shown by equation (16), the time domain response of the dielectric properties of the mixture of cells has two characteristic time constants $\tau_{1}$ and $\tau_{2}$. The first time constant, $\tau_{1}$, characterizes the cell membrane. The second time constant, $\tau_{2}$, characterizes the polarization of the interior of the cell (cytoplasm) with respect to the extracellular phase (suspending medium). These two time constants were characterized in Pauly and Schwan's paper [9], which was based on a frequency domain analysis of Maxwell's mixture theory [1]. In their paper, they adopted a simplified model based on three approximations.

(a) The conductivity of the cell membrane was considered to be very small in comparison with the cytoplasm and the suspending medium $\left(\sigma_{\mathrm{mem}} \ll \sigma_{\mathrm{m}}\right.$ and $\left.\sigma_{\mathrm{i}}\right)$. This enables some terms containing $\sigma_{\text {mem }}$ to be neglected.

(b) It was assumed that the membrane thickness is small when compared with the particle radius $(d \ll R)$. This lead to the approximation

$$
\gamma^{3}=\left(\frac{R+d}{R}\right)^{3} \approx 1+3 \frac{d}{R} .
$$

(c) The displacement current in the suspending medium and the cytoplasm is negligible compared with the conduction current. This leads to the assumption that the suspending medium and cytoplasm are pure conductors, which is only valid at frequencies much lower than the charge relaxation frequency of the system, leading to

$$
\varepsilon_{\mathrm{m}}=\varepsilon_{\mathrm{i}}=0
$$

Taken together, these three approximations make $\tau_{1}$ independent of the permittivity of the suspending medium and the cytoplasm and $\tau_{2}$ independent of the permittivity and conductivity of the membrane.

Using equations (18) and (19) in our model and expanding equations (16a) and (16b), we obtain (see details in appendix II)

$$
\begin{gathered}
\tau_{1}=\frac{R \varepsilon_{\mathrm{mem}}\left[(2+\varphi) \sigma_{\mathrm{m}}+(1-\varphi) \sigma_{\mathrm{i}}\right]}{(2+\varphi) \sigma_{\mathrm{m}}\left(d \sigma_{\mathrm{i}}+R \sigma_{\mathrm{mem}}\right)+R(1-\varphi) \sigma_{\mathrm{i}} \sigma_{\mathrm{mem}}}, \\
\tau_{2}=\frac{(1-\varphi) \varepsilon_{\mathrm{i}}+(2+\varphi) \varepsilon_{\mathrm{m}}}{(1-\varphi) \sigma_{\mathrm{i}}+(2+\varphi) \sigma_{\mathrm{m}}}
\end{gathered}
$$

Introducing the general expression for the specific membrane capacitance $C_{\mathrm{mem}}=\varepsilon_{\mathrm{mem}} / d$ and specific membrane conductance $G_{\mathrm{mem}}=\sigma_{\mathrm{mem}} / d$, then equation (20a) becomes the well-known time constant expression given by Pauly and Schwan [9]:

$$
\tau_{1}=R C_{\text {mem }} \frac{\frac{1}{\sigma_{\mathrm{i}}}+\left(\frac{1-\varphi}{2+\varphi}\right) \frac{1}{\sigma_{\mathrm{m}}}}{1+R G_{\mathrm{mem}}\left[\frac{1}{\sigma_{\mathrm{i}}}+\left(\frac{1-\varphi}{2+\varphi}\right) \frac{1}{\sigma_{\mathrm{m}}}\right]} .
$$

For the low volume fraction case, $\varphi<<1$, equations (21) and (20b) become

$$
\begin{aligned}
\tau_{1} & =R C_{\mathrm{mem}} \frac{\frac{1}{\sigma_{\mathrm{i}}}+\frac{1}{2 \sigma_{\mathrm{m}}}}{1+R G_{\mathrm{mem}}\left(\frac{1}{\sigma_{\mathrm{i}}}+\frac{1}{2 \sigma_{\mathrm{m}}}\right)}, \\
\tau_{2} & =\frac{\varepsilon_{\mathrm{i}}+2 \varepsilon_{\mathrm{m}}}{\sigma_{\mathrm{i}}+2 \sigma_{\mathrm{m}}} .
\end{aligned}
$$


Equation (22a) is the simplified form of the expression for the time constant $\tau_{1}$ for small volume fractions. This expression was also derived by Kotnik et al [21] by applying the Laplace transformation to an expression for the transmembrane voltage, derived for a spherical cell in an infinite homogeneous electric field. In this paper, our analysis includes the effect of the volume fraction, using Maxwell's mixture equation, to represent measurements of real systems. Equation (22b) is the Maxwell-Wagner relaxation time constant for a single homogeneous sphere suspended in a homogeneous medium, due to the accumulation of free charge at the surface of the sphere [23].

By contrast to Pauly and Schwan's model, the three approximations outlined above are not required for our analytical model. Compared with the derivation of Kotnik et al [21], the inclusion of the volume fraction into our model makes it amenable for the analysis of the dielectric properties of particles in suspension. The special case of zero volume fraction $(\varphi=0)$, no particle in the suspending medium, is analysed in appendix III.

\subsection{Time domain dielectric spectroscopy analysis}

According to equation (14), the dielectric response of the permittivity of a mixture in the time domain can be separated into three parts.

(i) The instantaneous response.

The instantaneous response (at $t=0$ ) is given by

$$
\left.\varepsilon_{\text {mix }}(t)\right|_{t=0}=\frac{\varepsilon_{\mathrm{m}} a_{1}}{b_{1}} .
$$

For small volume fraction $\varphi<<1$, we neglect terms containing $\varphi$, leading to $a_{1} / b_{1}=1$, so that

$$
\left.\varepsilon_{\text {mix }}(t)\right|_{t=0}=\varepsilon_{\mathrm{m}} .
$$

Therefore, the instantaneous response is dominated by the permittivity of the suspending medium.

(ii) The steady-state response.

The steady-state response is given by the term $k_{1} U_{0}(t)$, which is non-zero for all times except $t=0$. When time tends to infinity, the response of the dielectric properties of the mixture becomes

$$
\left.\varepsilon_{\text {mix }}(t)\right|_{t \rightarrow \infty}=k_{1}=\frac{\sigma_{\mathrm{m}} a_{3}}{b_{3}} .
$$

Following the same argument as above for small volume fractions $\varphi<<1$, we neglect terms containing $\varphi$, to give $a_{3} / b_{3}=1$, so that

$$
\left.\varepsilon_{\text {mix }}(t)\right|_{t \rightarrow \infty}=\sigma_{\mathrm{m}} .
$$

This shows that the steady-state response is governed by the conductivity of the suspending medium.

(iii) Charging exponential response (intermediate times).

The charging (exponential) response is related to the two time constant terms: $k_{2} \mathrm{e}^{-t / \tau_{1}} U_{0}(t)$ and $k_{3} \mathrm{e}^{-t / \tau_{2}} U_{0}(t)$. The dielectric dispersion of each characteristic relaxation time constant can be derived in the time domain as

$$
\begin{aligned}
\Delta \varepsilon_{t_{1}} & =k_{2}, \\
\Delta \varepsilon_{t_{2}} & =k_{3} .
\end{aligned}
$$

It can be seen that these dielectric decrements are independent of time and are defined by the properties of the cell and medium.

\subsection{Frequency domain dielectric spectroscopy analysis}

The time domain response can be converted back into the frequency domain by Fourier transform of equation (16) to give

$$
\tilde{\varepsilon}_{\text {mix }}(\omega)=\frac{\varepsilon_{\mathrm{m}} a_{1}}{b_{1}}+\frac{k_{2} \tau_{1}}{1+j \omega \tau_{1}}+\frac{k_{3} \tau_{2}}{1+j \omega \tau_{2}}+\frac{k_{1}}{j \omega} .
$$

From equation (29), we have

$$
\begin{aligned}
\tilde{\varepsilon}_{\text {mix }}(\infty) & =\frac{\varepsilon_{\mathrm{m}} a_{1}}{b_{1}}, \\
\Delta \varepsilon_{\omega_{1}} & =k_{2} \tau_{1}, \\
\Delta \varepsilon_{\omega_{2}} & =k_{3} \tau_{2}, \\
\sigma_{0} & =k_{1} .
\end{aligned}
$$

Equation (30) gives the limiting high frequency complex permittivity; $\Delta \varepsilon_{\omega}$ is the magnitude of the dielectric dispersions, with characteristic relaxation time constant $\tau$; and $\sigma_{0}$ is the limiting low frequency conductivity. It can be seen that $\Delta \varepsilon_{\omega}$ and $\tau$ are all frequency independent positive numbers [24,25].

By separating the real and imaginary parts of equation (29), we have

$$
\begin{aligned}
\tilde{\varepsilon}_{\text {mix }}(\omega)= & \frac{\varepsilon_{\mathrm{m}} a_{1}}{b_{1}}+\frac{k_{2} \tau_{1}}{1+\omega^{2} \tau_{1}^{2}}+\frac{k_{3} \tau_{2}}{1+\omega^{2} \tau_{2}^{2}}-j \\
& \times\left(\frac{k_{1}}{\omega}+\frac{\omega k_{2} \tau_{1}^{2}}{1+\omega^{2} \tau_{1}^{2}}+\frac{\omega k_{3} \tau_{2}^{2}}{1+\omega^{2} \tau_{2}^{2}}\right) .
\end{aligned}
$$

Comparing the format of equations (4) and (34), it can be seen that the recovered relative permittivity and conductivity of the mixture is obtained from the real and imaginary part, respectively:

$$
\begin{aligned}
& \varepsilon_{\text {mix }}(\omega)=\frac{1}{\varepsilon_{0}}\left(\frac{\varepsilon_{\mathrm{m}} a_{1}}{b_{1}}+\frac{k_{2} \tau_{1}}{1+\omega^{2} \tau_{1}^{2}}+\frac{k_{3} \tau_{2}}{1+\omega^{2} \tau_{2}^{2}}\right), \\
& \sigma_{\text {mix }}(\omega)=\omega\left(\frac{k_{1}}{\omega}+\frac{\omega k_{2} \tau_{1}^{2}}{1+\omega^{2} \tau_{1}^{2}}+\frac{\omega k_{3} \tau_{2}^{2}}{1+\omega^{2} \tau_{2}^{2}}\right) .
\end{aligned}
$$

We now compare the recovered dielectric spectrum (permittivity and conductivity) with the original mixture equation (equation (1)) for two cases: low $\left(\sigma_{\mathrm{m}}=0.016 \mathrm{~S} \mathrm{~m}^{-1}\right)$ and high $\left(\sigma_{\mathrm{m}}=1.6 \mathrm{~S} \mathrm{~m}^{-1}\right)$ suspending medium conductivity. Other parameters are $\varepsilon_{o}=8.854 \times 10^{-12} \mathrm{Fm}^{-1}, R=3 \times$ $10^{-6} \mathrm{~m}, d=5 \times 10^{-9} \mathrm{~m}, \varepsilon_{\mathrm{m}}=80 \times \varepsilon_{\mathrm{o}}, \varepsilon_{\mathrm{mem}}=5 \times \varepsilon_{\mathrm{o}}$, $\sigma_{\text {mem }}=10^{-8} \mathrm{~S} \mathrm{~m}^{-1}, \varepsilon_{\mathrm{i}}=60 \times \varepsilon_{\mathrm{o}}, \sigma_{\mathrm{i}}=0.4 \mathrm{~S} \mathrm{~m}^{-1}$ and volume fraction $\varphi=0.0098 \ll 1$.

Figure 2 shows a comparison of the relative permittivity and conductivity spectra derived from the mixture equation (real and imaginary part of equation (1)) and the recovered equations (equations (35) and (36)). It is clear that there is perfect agreement between both approaches, validating that equations (34) is an equivalent form of Maxwell's mixture equation (equation (1)), written in terms of characteristic relaxation time constants. 

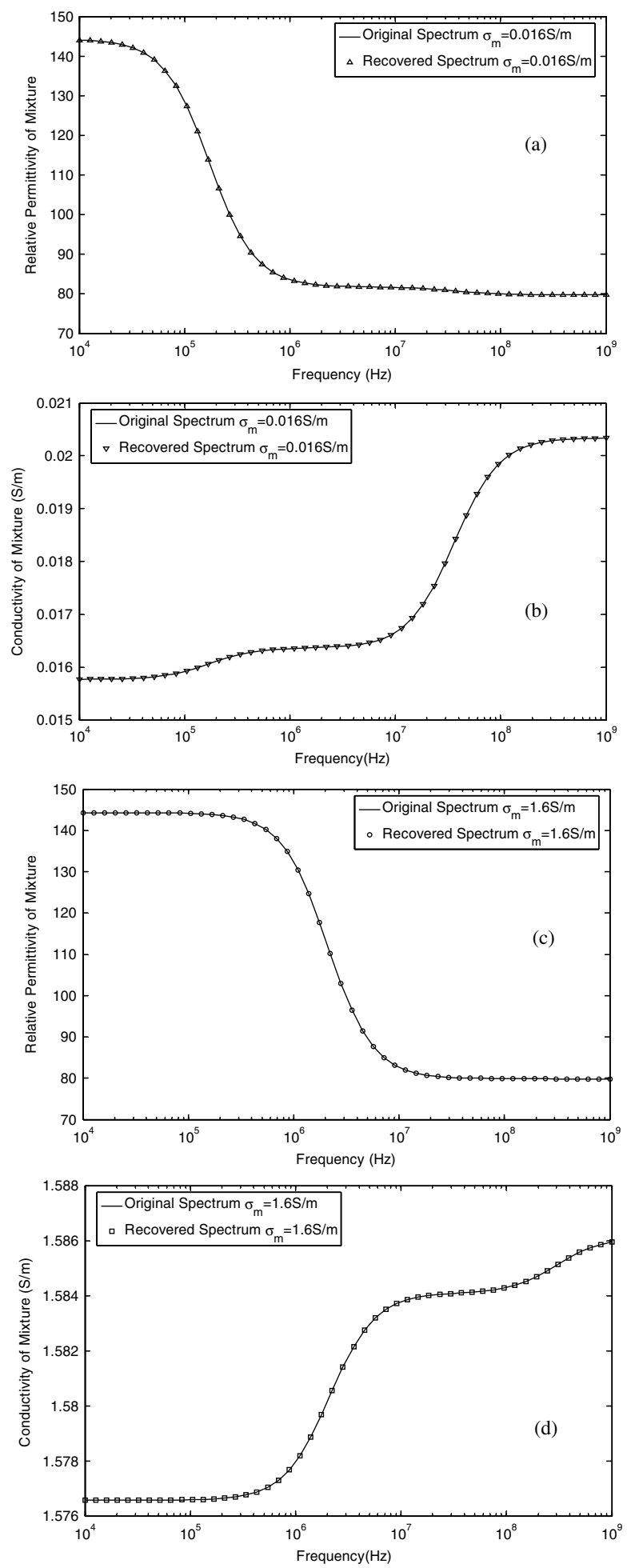

Figure 2. (a) Comparison of the frequency dependent relative permittivity derived from the original $(-)$ and recovered $(\triangle)$ mixture equation for low conductivity suspending medium. (b) Comparison of the frequency dependent conductivity derived from the original $(-)$ and recovered $(\nabla)$ mixture equation for low conductivity suspending medium. (c) Comparison of the frequency dependent relative permittivity derived from the original ( - ) and recovered $(O)$ mixture equation for high conductivity suspending medium. (d) Comparison of the frequency dependent conductivity derived from the original ( $\longrightarrow$ ) and recovered ( $\square$ ) mixture equation for high conductivity suspending medium.

\subsection{Relationship between time domain and frequency domain}

From the above expression we derive equations for the dielectric permittivity in both time and frequency domains. Let us examine the relationship between these two domains.

For equations (25) and (30), we have

$$
\left.\varepsilon_{\text {mix }}(t)\right|_{t=0}=\frac{\varepsilon_{\mathrm{m}} a_{1}}{b_{1}}=\tilde{\varepsilon}_{\text {mix }}(\infty) .
$$

Equation (37) relates the instantaneous response of the complex permittivity of the mixture in the time domain to the high frequency limit in the frequency domain. At high frequencies the charge movement (which creates the induced dipoles) is fast and can only be observed in a very short time window. When $t=0$, the instantaneous response of the mixture is determined by the initial energy storage capacity (or charge accumulation) of the system. This is dominated by the permittivity of the suspending medium. As time goes on, the charges are set into motion by the external field, and the response of the system begins to be dominated by the conductivity of the system. This is a measure of the ease with which charge moves through a material [26].

Correspondingly, from equations (26) and (33), we have

$$
\left.\varepsilon_{\text {mix }}(t)\right|_{t \rightarrow \infty}=\frac{\sigma_{\mathrm{m}} a_{3}}{b_{3}}=\sigma_{0} .
$$

Equation (38) relates the steady-state response of the complex permittivity of the mixture in the time domain to the low frequency limit in the frequency domain. At low frequencies, movement of charge (to create the dipole) is slower and can be observed over a relatively long time period. While time tends to infinity, the total response of the system is determined by the frequency independent part of the conductivity (due to ionic conduction), which is dominated by the conductivity of the suspending medium.

The dielectric dispersions in the time and frequency domains (equation (27) with (31) and equation (28) with (32)) are directly linked by the Fourier transform, shown in equation (39):

$$
\mathrm{e}^{-t / \tau} U_{0}(t) \leftrightarrow \frac{\tau}{1+j \omega \tau}
$$

Figure 3 presents a simulation of the time-dependent permittivity of the mixture (equation (14)) using the same parameters as previously for a high conductivity suspending medium $\left(\sigma_{\mathrm{m}}=1.6 \mathrm{~S} \mathrm{~m}^{-1}\right)$. It can be seen that the dispersion in permittivity in the short time period corresponds to the relaxation process at high frequency, as the membrane is short-circuited. The dispersion in permittivity at long time corresponds to the relaxation process for the cell membrane, which occurs at low frequency. This result indicates that it is possible to derive system information in the frequency domain by analysing the behaviour of the system in the time domain first. For example the time-dependent response of the system could be probed with a short duration pulse or a noise excitation and the frequency response of the system recovered using digital signal processing (DSP) techniques such as fast Fourier transform (FFT). 


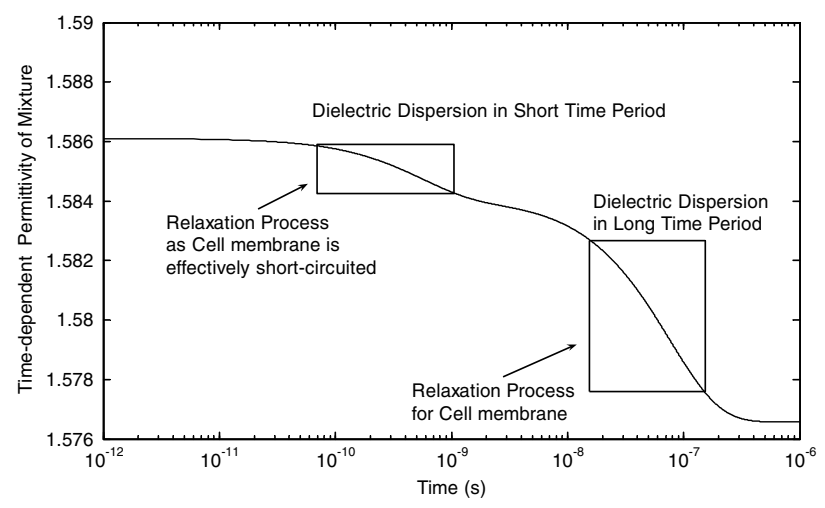

Figure 3. Plot of the time-dependent Maxwell's mixture equation (equation (14)) of cells as a function of time (see text for further details).

\section{Conclusion}

In this paper, we have presented a time domain expression for Maxwell's mixture equation using the Laplace transformation. As an example, we have analysed the dielectric properties of a single shelled spherical cell in suspension both in the time domain and the frequency domain. The full analytical expressions of the two characteristic relaxation time constants have been derived. Our method can be extended to the multishelled model and high concentration cases using Hanai's equation [15-17]. The model presented here is helpful in the determination of the behaviour of single particles in time-varying electric fields and transient response in general. The link between dielectric spectroscopy in the time and frequency domains is clearly illustrated. Future work will apply the time domain mixture equation to the analysis of the time-dependent dielectric response of our micro-impedance systems.

\section{Acknowledgments}

This work was partly funded by the University of Southampton Life Sciences Initiative (LSI). The authors would like to thank Dr Kotnik for useful discussions. SG would like to acknowledge the financial contribution of the Fond National Suisse (FNS) Bourse Pour Jeunes Chercheurs.

\section{Appendix I}

The coefficients $A$ and $B$ are

$$
\begin{aligned}
A= & s\left[\gamma^{3}\left(\varepsilon_{\mathrm{i}}+2 \varepsilon_{\mathrm{mem}}\right)+2\left(\varepsilon_{\mathrm{i}}-\varepsilon_{\mathrm{mem}}\right)\right]+\gamma^{3}\left(\sigma_{\mathrm{i}}+2 \sigma_{\mathrm{mem}}\right) \\
& +2\left(\sigma_{\mathrm{i}}-\sigma_{\mathrm{mem}}\right), \\
B= & s\left[\gamma^{3}\left(\varepsilon_{\mathrm{i}}+2 \varepsilon_{\mathrm{mem}}\right)-\left(\varepsilon_{\mathrm{i}}-\varepsilon_{\mathrm{mem}}\right)\right]+\gamma^{3}\left(\sigma_{\mathrm{i}}+2 \sigma_{\mathrm{mem}}\right) \\
& -2\left(\sigma_{\mathrm{i}}-\sigma_{\mathrm{mem}}\right) .
\end{aligned}
$$

The coefficients $C$ and $D$ are

$$
\begin{aligned}
& C=A\left(\varepsilon_{\mathrm{mem}} s+\sigma_{\mathrm{mem}}\right)+2 B\left(\varepsilon_{\mathrm{m}} s+\sigma_{\mathrm{m}}\right), \\
& D=A\left(\varepsilon_{\mathrm{mem}} s+\sigma_{\mathrm{mem}}\right)-B\left(\varepsilon_{\mathrm{m}} s+\sigma_{\mathrm{m}}\right) .
\end{aligned}
$$

Substitute the expressions for $A$ and $B$ into equations (AI.3) and (AI.4) and the expressions for $C$ and $D$ are

$$
\begin{aligned}
& C= \\
& s^{2}\left[\gamma^{3}\left(\varepsilon_{\mathrm{i}}+2 \varepsilon_{\mathrm{mem}}\right)\left(\varepsilon_{\mathrm{mem}}+2 \varepsilon_{\mathrm{m}}\right)+2\left(\varepsilon_{\mathrm{i}}-\varepsilon_{\mathrm{mem}}\right)\left(\varepsilon_{\mathrm{mem}}-\varepsilon_{\mathrm{m}}\right)\right] \\
&+s \gamma^{3}\left[\left(\varepsilon_{\mathrm{i}}+2 \varepsilon_{\mathrm{mem}}\right)\left(\sigma_{\mathrm{mem}}+2 \sigma_{\mathrm{m}}\right)+\left(\sigma_{\mathrm{i}}+2 \sigma_{\mathrm{mem}}\right)\left(\varepsilon_{\mathrm{mem}}+2 \varepsilon_{\mathrm{m}}\right)\right] \\
&+2 s\left[\left(\sigma_{\mathrm{mem}}-\sigma_{\mathrm{m}}\right)\left(\varepsilon_{\mathrm{i}}-\varepsilon_{\mathrm{mem}}\right)+\left(\sigma_{\mathrm{i}}-\sigma_{\mathrm{mem}}\right)\left(\varepsilon_{\mathrm{mem}}-\varepsilon_{\mathrm{m}}\right)\right] \\
&+\gamma^{3}\left(\sigma_{\mathrm{i}}+2 \sigma_{\mathrm{mem}}\right)\left(\sigma_{\mathrm{mem}}+2 \sigma_{\mathrm{m}}\right)+2\left(\sigma_{\mathrm{i}}-\sigma_{\mathrm{mem}}\right)\left(\sigma_{\mathrm{mem}}-\sigma_{\mathrm{m}}\right), \\
& D=\quad(\mathrm{AI} .5) \\
& s^{2}\left[\gamma^{3}\left(\varepsilon_{\mathrm{i}}+2 \varepsilon_{\mathrm{mem}}\right)\left(\varepsilon_{\mathrm{mem}}-\varepsilon_{\mathrm{m}}\right)+\left(\varepsilon_{\mathrm{i}}-\varepsilon_{\mathrm{mem}}\right)\left(2 \varepsilon_{\mathrm{mem}}+\varepsilon_{\mathrm{m}}\right)\right] \\
&+s \gamma^{3}\left[\left(\varepsilon_{\mathrm{i}}+2 \varepsilon_{\mathrm{mem}}\right)\left(\sigma_{\mathrm{mem}}-\sigma_{\mathrm{m}}\right)+\left(\sigma_{\mathrm{i}}+2 \sigma_{\mathrm{mem}}\right)\left(\varepsilon_{\mathrm{mem}}-\varepsilon_{\mathrm{m}}\right)\right] \\
&+s\left[\left(2 \sigma_{\mathrm{mem}}+\sigma_{\mathrm{m}}\right)\left(\varepsilon_{\mathrm{i}}-\varepsilon_{\mathrm{mem}}\right)+\left(\sigma_{\mathrm{i}}-\sigma_{\mathrm{mem}}\right)\left(2 \varepsilon_{\mathrm{mem}}+\varepsilon_{\mathrm{m}}\right)\right] \\
&+\gamma^{3}\left(\sigma_{\mathrm{i}}+2 \sigma_{\mathrm{mem}}\right)\left(\sigma_{\mathrm{mem}}-\sigma_{\mathrm{m}}\right)+\left(\sigma_{\mathrm{i}}-\sigma_{\mathrm{mem}}\right)\left(2 \sigma_{\mathrm{mem}}+\sigma_{\mathrm{m}}\right) .
\end{aligned}
$$

Substitute equations (AI.5) and (AI.6) into equation (8) and expand in decreasing order of $s$ to obtain the coefficients $a_{n}$ and $b_{n}(n=1,2,3)$ :

$$
\begin{aligned}
a_{1}= & \gamma^{3}\left(\varepsilon_{\mathrm{i}}+2 \varepsilon_{\mathrm{mem}}\right)\left[\left(\varepsilon_{\mathrm{mem}}+2 \varepsilon_{\mathrm{m}}\right)+2 \varphi\left(\varepsilon_{\mathrm{mem}}-\varepsilon_{\mathrm{m}}\right)\right] \\
& +2\left(\varepsilon_{\mathrm{i}}-\varepsilon_{\mathrm{mem}}\right)\left[\left(\varepsilon_{\mathrm{mem}}-\varepsilon_{\mathrm{m}}\right)+\varphi\left(2 \varepsilon_{\mathrm{mem}}+\varepsilon_{\mathrm{m}}\right)\right], \\
a_{2}= & \gamma^{3}\left(\varepsilon_{\mathrm{i}}+2 \varepsilon_{\mathrm{mem}}\right)\left[\left(\sigma_{\mathrm{mem}}+2 \sigma_{\mathrm{m}}\right)+2 \varphi\left(\sigma_{\mathrm{mem}}-\sigma_{\mathrm{m}}\right)\right. \\
& +\gamma^{3}\left(\sigma_{\mathrm{i}}+2 \sigma_{\mathrm{mem}}\right)\left[\left(\varepsilon_{\mathrm{mem}}+2 \varepsilon_{\mathrm{m}}\right)+2 \varphi\left(\varepsilon_{\mathrm{mem}}-\varepsilon_{\mathrm{m}}\right)\right] \\
& +2\left(\varepsilon_{\mathrm{i}}-\varepsilon_{\mathrm{mem}}\right)\left[\left(\sigma_{\mathrm{mem}}-\sigma_{\mathrm{m}}\right)+\varphi\left(2 \sigma_{\mathrm{mem}}+\sigma_{\mathrm{m}}\right)\right] \\
& +2\left(\sigma_{\mathrm{i}}-\sigma_{\mathrm{mem}}\right)\left[\left(\varepsilon_{\mathrm{mem}}-\varepsilon_{\mathrm{m}}\right)+\varphi\left(2 \varepsilon_{\mathrm{mem}}+\varepsilon_{\mathrm{m}}\right)\right],
\end{aligned}
$$

$$
\begin{aligned}
a_{3}= & \gamma^{3}\left(\sigma_{\mathrm{i}}+2 \sigma_{\mathrm{mem}}\right)\left[\sigma_{\mathrm{mem}}(1+2 \varphi)+2 \sigma_{\mathrm{m}}(1-\varphi)\right] \\
& +2\left(\sigma_{\mathrm{i}}-\sigma_{\mathrm{mem}}\right)\left[\sigma_{\mathrm{mem}}(1+2 \varphi)-\sigma_{\mathrm{m}}(1-\varphi)\right],
\end{aligned}
$$

$$
\begin{aligned}
b_{1}= & \gamma^{3}\left(\varepsilon_{\mathrm{i}}+2 \varepsilon_{\mathrm{mem}}\right)\left[\left(\varepsilon_{\mathrm{mem}}+2 \varepsilon_{\mathrm{m}}\right)-\varphi\left(\varepsilon_{\mathrm{mem}}-\varepsilon_{\mathrm{m}}\right)\right] \\
& +\left(\varepsilon_{\mathrm{i}}-\varepsilon_{\mathrm{mem}}\right)\left[2\left(\varepsilon_{\mathrm{mem}}-\varepsilon_{\mathrm{m}}\right)-\varphi\left(2 \varepsilon_{\mathrm{mem}}+\varepsilon_{\mathrm{m}}\right)\right],
\end{aligned}
$$

$$
\begin{aligned}
b_{2}= & \gamma^{3}\left(\varepsilon_{\mathrm{i}}+2 \varepsilon_{\mathrm{mem}}\right)\left[\left(\sigma_{\mathrm{mem}}+2 \sigma_{\mathrm{m}}\right)-\varphi\left(\sigma_{\mathrm{mem}}-\sigma_{\mathrm{m}}\right)\right] \\
& +\gamma^{3}\left(\sigma_{\mathrm{i}}+2 \sigma_{\mathrm{mem}}\right)\left[\left(\varepsilon_{\mathrm{mem}}+2 \varepsilon_{\mathrm{m}}\right)-\varphi\left(\varepsilon_{\mathrm{mem}}-\varepsilon_{\mathrm{m}}\right)\right] \\
& +\left(\varepsilon_{\mathrm{i}}-\varepsilon_{\mathrm{mem}}\right)\left[2\left(\sigma_{\mathrm{mem}}-\sigma_{\mathrm{m}}\right)-\varphi\left(2 \sigma_{\mathrm{mem}}+\sigma_{\mathrm{m}}\right)\right] \\
& +\left(\sigma_{\mathrm{i}}-\sigma_{\mathrm{mem}}\right)\left[2\left(\varepsilon_{\mathrm{mem}}-\varepsilon_{\mathrm{m}}\right)-\varphi\left(2 \varepsilon_{\mathrm{mem}}+\varepsilon_{\mathrm{m}}\right)\right],
\end{aligned}
$$

$$
\begin{aligned}
b_{3}= & \gamma^{3}\left(\sigma_{\mathrm{i}}+2 \sigma_{\mathrm{mem}}\right)\left[\sigma_{\mathrm{mem}}(1-\varphi)+\sigma_{\mathrm{m}}(2+\varphi)\right] \\
& +\left(\sigma_{\mathrm{i}}-\sigma_{\mathrm{mem}}\right)\left[2 \sigma_{\mathrm{mem}}(1-\varphi)-\sigma_{\mathrm{m}}(2+\varphi)\right] .
\end{aligned}
$$

The time domain expression for the complex conductivity can be solved in a similar way to the expression for the complex permittivity (equation (14)). We use the relationship between the complex permittivity and conductivity (equation (17)) in the following procedure.

Combine equations (17) and (9),

$$
\begin{aligned}
\tilde{\sigma}_{\text {mix }}(s) & =d_{1} s+d_{2}+\frac{d_{3} s+d_{4}}{b_{1} s^{2}+b_{2} s+b_{3}} \\
& =d_{1} s+d_{2}+\frac{d_{3}\left(s-s_{7}\right)}{b_{1}\left(s-s_{3}\right)\left(s-s_{4}\right)},
\end{aligned}
$$


where the coefficients $d_{n}(n=1,2,3,4)$ are given by

$$
\begin{aligned}
d_{1} & =\frac{\varepsilon_{\mathrm{m}} a_{1}}{b_{1}}, \\
d_{2} & =\frac{c_{1}}{b_{1}}, \\
d_{3} & =c_{2}-d_{2} b_{2}, \\
d_{4} & =c_{3}-d_{2} b_{3}
\end{aligned}
$$

and coefficient $s_{7}$ is given by

$$
s_{7}=-\frac{d_{4}}{d_{3}} .
$$

Therefore, the time domain expression of $\sigma_{\text {mix }}(t)$ is given by

$$
\sigma_{\text {mix }}(t)=d_{1} \delta^{\prime}(t)+d_{2} \delta(t)+\left(k_{4} \mathrm{e}^{-t / \tau_{1}}+k_{5} \mathrm{e}^{-t / \tau_{2}}\right) U_{0}(t),
$$

where $\delta^{\prime}(t)$ is the time derivative of $\delta(t)$ and the coefficients $k_{4}$ and $k_{5}$ are given by

$$
\begin{aligned}
& k_{4}=\frac{d_{3}\left(s_{3}-s_{7}\right)}{b_{1}\left(s_{3}-s_{4}\right)}, \\
& k_{5}=\frac{d_{3}\left(s_{4}-s_{7}\right)}{b_{1}\left(s_{4}-s_{3}\right)} .
\end{aligned}
$$

\section{Appendix II}

The two time constants are determined by the three coefficients $b_{1}, b_{2}$ and $b_{3}$. According to the three assumptions in section 3.1, we can simplify these three coefficients as below.

By expanding the full equation (AI.11), we obtain

$$
\begin{aligned}
b_{1}= & \gamma^{3}\left(\varepsilon_{\mathrm{i}} \varepsilon_{\mathrm{mem}}+2 \varepsilon_{\mathrm{i}} \varepsilon_{\mathrm{m}}-\varphi \varepsilon_{\mathrm{i}} \varepsilon_{\mathrm{mem}}+\varphi \varepsilon_{\mathrm{m}} \varepsilon_{\mathrm{i}}+2 \varepsilon_{\mathrm{mem}}^{2}\right. \\
& \left.+4 \varepsilon_{\mathrm{mem}} \varepsilon_{\mathrm{m}}-2 \varphi \varepsilon_{\mathrm{mem}}^{2}+2 \varphi \varepsilon_{\mathrm{mem}} \varepsilon_{\mathrm{m}}\right) \\
& +\left(2 \varepsilon_{\mathrm{i}} \varepsilon_{\mathrm{mem}}-2 \varepsilon_{\mathrm{i}} \varepsilon_{\mathrm{m}}-2 \varphi \varepsilon_{\mathrm{i}} \varepsilon_{\mathrm{mem}}-\varphi \varepsilon_{\mathrm{m}} \varepsilon_{\mathrm{i}}-2 \varepsilon_{\mathrm{mem}}^{2}\right. \\
& \left.+2 \varepsilon_{\mathrm{mem}} \varepsilon_{\mathrm{m}}+2 \varphi \varepsilon_{\mathrm{mem}}^{2}+\varphi \varepsilon_{\mathrm{mem}} \varepsilon_{\mathrm{m}}\right) .
\end{aligned}
$$

Substitute equation (18) into equation (AII.1) and since $d \ll R$ ignore terms $d / R$ :

$$
b_{1}=3 \varepsilon_{\mathrm{mem}}\left[(1-\varphi) \varepsilon_{\mathrm{i}}+(\varphi+2) \varepsilon_{\mathrm{m}}\right] \text {. }
$$

Substitute equation (23) into equation (AI.11) and then expand the full equation, ignoring all terms containing $\sigma_{\text {mem }}\left(\sigma_{\text {mem }} \ll\right.$ $\sigma_{\mathrm{m}}$ and $\left.\sigma_{\mathrm{i}}\right)$ :

$$
\begin{aligned}
b_{2}= & \varepsilon_{\mathrm{mem}}\left\{\gamma^{3}\left[(4+2 \varphi) \sigma_{\mathrm{m}}+(1-\varphi) \sigma_{\mathrm{i}}\right]+(2+\varphi) \sigma_{\mathrm{m}}\right. \\
& \left.+2(1-\varphi) \sigma_{\mathrm{i}}\right\} .
\end{aligned}
$$

Substitute equation (18) into equation (AII.3):

$$
\begin{aligned}
b_{2}= & \varepsilon_{\mathrm{mem}}\left\{3\left[(2+\varphi) \sigma_{\mathrm{m}}+(1-\varphi) \sigma_{\mathrm{i}}\right]+3 \frac{d}{R}\left[(4+2 \varphi) \sigma_{\mathrm{m}}\right.\right. \\
& \left.\left.+(1-\varphi) \sigma_{\mathrm{i}}\right]\right\}
\end{aligned}
$$

Ignore terms with $d / R$ :

$$
b_{2}=3 \varepsilon_{\mathrm{mem}}\left[(2+\varphi) \sigma_{\mathrm{m}}+(1-\varphi) \sigma_{\mathrm{i}}\right] .
$$

Substitute equation (18) into equation (AI.12) and ignore terms with $d / R$ :

$$
\begin{aligned}
b_{3}= & \sigma_{\mathrm{mem}}(1-\varphi)\left[\left(1+3 \frac{d}{R}\right)\left(\sigma_{\mathrm{i}}+2 \sigma_{\mathrm{mem}}\right)+2\left(\sigma_{\mathrm{i}}-\sigma_{\mathrm{mem}}\right)\right] \\
& +\sigma_{m}(2+\varphi)\left[\left(1+3 \frac{d}{R}\right)\left(\sigma_{\mathrm{i}}+2 \sigma_{\mathrm{mem}}\right)-\left(\sigma_{\mathrm{i}}-\sigma_{\mathrm{mem}}\right)\right] \\
= & 3 \sigma_{\mathrm{mem}}(1-\varphi)\left[\sigma_{\mathrm{i}}+\frac{d}{R}\left(\sigma_{\mathrm{i}}+2 \sigma_{\mathrm{mem}}\right)\right]+3 \sigma_{\mathrm{m}}(2+\varphi) \\
& \times\left[\sigma_{\mathrm{mem}}+\frac{d}{R}\left(\sigma_{\mathrm{i}}+2 \sigma_{\mathrm{mem}}\right)\right] \\
= & 3 \sigma_{\mathrm{mem}} \sigma_{\mathrm{i}}(1-\varphi)+3 \sigma_{\mathrm{m}}(2+\varphi)\left(\sigma_{\mathrm{mem}}+\frac{d}{R} \sigma_{\mathrm{i}}\right)
\end{aligned}
$$

(AII.6a)

$=3 \sigma_{\mathrm{mem}}\left[(2+\varphi) \sigma_{\mathrm{m}}+(1-\varphi) \sigma_{\mathrm{i}}\right]$

Combining equations (AII.2), (AII.5) and (AII.6b) and considering $\sigma_{\text {mem }} \ll \sigma_{\mathrm{m}}$ and $\sigma_{\mathrm{i}}$, we have

$$
\frac{b_{2}^{2}}{4 b_{1} b_{3}}=\frac{\varepsilon_{\mathrm{mem}}\left[(2+\varphi) \sigma_{\mathrm{m}}+(1-\varphi) \sigma_{\mathrm{i}}\right]}{4 \sigma_{\mathrm{mem}}\left[(2+\varphi) \varepsilon_{\mathrm{m}}+(1-\varphi) \varepsilon_{\mathrm{i}}\right]} \gg 1
$$

Equation (AII.7) implies

$$
b_{2}^{2} \gg 4 b_{1} b_{3} .
$$

For the first time constant $\tau_{1}$, equation (16a) is rewritten as

$$
\tau_{1}=\frac{b_{2}+\sqrt{b_{2}^{2}-4 b_{1} b_{3}}}{2 b_{3}} \text {. }
$$

Considering equation (AII.8), equation (AII.9) becomes

$$
\tau_{1}=\frac{b_{2}}{b_{3}} .
$$

Combining equations (AII.5), (AII.6a) and (AII.10), the first time constant becomes

$$
\begin{aligned}
\tau_{1} & =\frac{\varepsilon_{\mathrm{mem}}\left[(2+\varphi) \sigma_{\mathrm{m}}+(1-\varphi) \sigma_{\mathrm{i}}\right]}{\sigma_{\mathrm{mem}} \sigma_{\mathrm{i}}(1-\varphi)+\sigma_{\mathrm{m}}(2+\varphi)\left(\sigma_{\mathrm{mem}}+\frac{d}{R} \sigma_{\mathrm{i}}\right)} \\
& =\frac{R \varepsilon_{\mathrm{mem}}\left[(2+\varphi) \sigma_{\mathrm{m}}+(1-\varphi) \sigma_{\mathrm{i}}\right]}{(2+\varphi) \sigma_{\mathrm{m}}\left(R \sigma_{\mathrm{mem}}+d \sigma_{\mathrm{i}}\right)+R(1-\varphi) \sigma_{\mathrm{i}} \sigma_{\mathrm{mem}}}
\end{aligned}
$$

For the second time constant $\tau_{2}$, from equation (AII.8), equation $(16 b)$ becomes

$$
\tau_{2}=\frac{b_{1}}{b_{2}}
$$

Therefore, combining equations (AII.2), (AII.5) and (AII.12), the second time constant $\tau_{2}$ becomes

$$
\begin{aligned}
\tau_{2} & =\frac{3 \varepsilon_{\mathrm{mem}}\left[(1-\varphi) \varepsilon_{\mathrm{i}}+(2+\varphi) \varepsilon_{\mathrm{m}}\right]}{3 \varepsilon_{\mathrm{mem}}\left[(2+\varphi) \sigma_{\mathrm{m}}+(1-\varphi) \sigma_{\mathrm{i}}\right]} \\
& =\frac{(1-\varphi) \varepsilon_{\mathrm{i}}+(2+\varphi) \varepsilon_{\mathrm{m}}}{(1-\varphi) \sigma_{\mathrm{i}}+(2+\varphi) \sigma_{\mathrm{m}}}
\end{aligned}
$$




\section{Appendix III}

For the special case of volume fraction $\varphi=0$, there is no particle in the medium: equations (AI.7)-(AI.12) become

$a_{1}=2\left(\varepsilon_{\mathrm{i}}-\varepsilon_{\mathrm{mem}}\right)\left(\varepsilon_{\mathrm{mem}}-\varepsilon_{\mathrm{m}}\right)$,

$a_{2}=2\left[\left(\varepsilon_{\mathrm{i}}-\varepsilon_{\mathrm{mem}}\right)\left(\sigma_{\mathrm{mem}}-\sigma_{\mathrm{m}}\right)+\left(\sigma_{\mathrm{i}}-\sigma_{\mathrm{mem}}\right)\left(\varepsilon_{\mathrm{mem}}-\varepsilon_{\mathrm{m}}\right)\right]$,

$a_{3}=2\left(\sigma_{\mathrm{i}}-\sigma_{\mathrm{mem}}\right)\left(\sigma_{\mathrm{mem}}-\sigma_{\mathrm{m}}\right)$,

$b_{1}=2\left(\varepsilon_{\mathrm{i}}-\varepsilon_{\mathrm{mem}}\right)\left(\varepsilon_{\mathrm{mem}}-\varepsilon_{\mathrm{m}}\right)$,

$b_{2}=2\left[\left(\varepsilon_{\mathrm{i}}-\varepsilon_{\mathrm{mem}}\right)\left(\sigma_{\mathrm{mem}}-\sigma_{\mathrm{m}}\right)+\left(\sigma_{\mathrm{i}}-\sigma_{\mathrm{mem}}\right)\left(\varepsilon_{\mathrm{mem}}-\varepsilon_{\mathrm{m}}\right)\right]$,

$b_{3}=2\left(\sigma_{\mathrm{i}}-\sigma_{\mathrm{mem}}\right)\left(\sigma_{\mathrm{mem}}-\sigma_{\mathrm{m}}\right)$.

Therefore $a_{n}=b_{n}(n=1,2,3)$, which means that the coefficients $c_{n}(n=1,2,3)$ (equations $\left.(12 a)-(12 c)\right)$ become

$$
\begin{aligned}
& c_{1}=\sigma_{\mathrm{m}} a_{1}, \\
& c_{2}=\sigma_{\mathrm{m}} a_{2}, \\
& c_{3}=\sigma_{\mathrm{m}} a_{3} .
\end{aligned}
$$

It is clear to see the relationship between the coefficients $a_{n}$, $b_{n}$ and $c_{n}$ :

$$
c_{n}=\sigma_{\mathrm{m}} a_{n}=\sigma_{\mathrm{m}} b_{n} .
$$

Using equation (AIII.8) in equations (10b) and (13), we obtain

$$
\begin{aligned}
& s_{3}=s_{5}, \\
& s_{4}=s_{6} .
\end{aligned}
$$

Equations (AIII.9a) and (AIII.9b) make $k_{2}=k_{3}=0$, which results in equation (34) being

$$
\tilde{\varepsilon}_{\text {mix }}(\omega)=\frac{\varepsilon_{\mathrm{m}} a_{1}}{b_{1}}-j \frac{k_{1}}{\omega}=\varepsilon_{\mathrm{m}}-j \frac{\sigma_{\mathrm{m}}}{\omega} .
$$

This shows that Maxwell's mixture equation returns to the expression for the complex permittivity of the medium, confirming the validity of the transformation between time and frequency domains.

\section{References}

[1] Maxwell J C 1954 A Treatise on Electricity and Magnetism (New York: Dover)

[2] Wagner K W 1914 Electricity of the dielectric behaviour on the basis of the Maxwell theory Arch. J. Elecktrotechn. 2 371-87

[3] Fricke H 1924 A mathematical treatment of the electrical conductivity of colloids and cell suspensions J. Gen. Physiol. 6 375-84

[4] Fricke H 1925 The electric capacity of suspensions with special reference to blood J. Gen. Physiol. 9 137-52

[5] Fricke $\mathrm{H}$ and Curtis H J 1935 The electric impedance of hemolyzed suspensions of mammalian erythrocytes J. Gen. Physiol. 18 821-36

[6] Cole K S 1928 Electric impedance of suspensions of spheres J. Gen. Physiol. 12 29-36
[7] Cole K S and Cole R H 1936 Electric impedance of asterias eggs J. Gen. Physiol. 19 609-23

[8] Cole K S and Curtis H J 1938 Electric impedance of single marine eggs J. Gen. Physiol. 21 591-9

[9] Pauly H and Schwan H P 1959 Uber die Impedanz einer Suspension von kugelformigen Teilchen mit einer Schale $Z$. Naturf. B 14125

[10] Schwan H P 1957 Electrical properties of tissues and cells $A d v$. Biol. Med. Phys. 5 147-209

[11] Schwan H P, Takashima S, Miyamoto V K and Stoeckenius W 1970 Electrical properties of phospholipid vesicles Biophys. J. 10 1102-19

[12] Foster K R and Schwan H P 1989 Dielectric properties of tissues and biological materials: a critical review Crit. Rev. Biomed. Eng. 17 25-104

[13] Asami K 2002 Characterization of heterogeneous systems by dielectric spectroscopy Prog. Polym. Sci. 27 1617-59

[14] Bruggeman D A G 1935 Berechnung verschiedener physikalischer Konstanten von heterogene Substanzen; I Dielektrizitätskonstanten und Leitfähigkeiten der Mischkörper aus isotropen Substanzen Ann. Phys. Lpz. 24 636-64

[15] Hanai T, Koizumi N and Irimajiri A 1975 A method for determining the dielectric constant and the conductivity of membrane-bounded particles of biological relevance Biophys. Struct. Mech. 1 285-94

[16] Hanai T and Koizumi N 1976 Numerical estimation on a theory of interfacial polarization developed for disperse systems in higher concentration Bull. Inst. Chem. Res. Kyoto Univ. $\mathbf{5 4} 248-54$

[17] Hanai T, Asami K and Koizumi N 1979 Dielectric theory of concentrated suspensions of shell-spheres in particular reference to the analysis of biological cell suspensions Bull. Inst. Chem. Res. Kyoto Univ. 57 297-305

[18] Schwan H P 1994 Electrical properties of tissues and cell suspensions: mechanisms and models Proc. 16th Annual Int. Conf. of IEEE Engineering in Medicine and Biology Society vol 1 pp 70-1

[19] Zhang H Z, Sekine K, Hanai T and Koizumi N 1983 Dielectric observations on polystyrene microcapsules and the theoretical analysis with reference to interfacial polarization Colloid Polym. Sci. 261 381-9

[20] Feldman Y, Ermolina I and Hayashi Y 2003 Time domain dielectric spectroscopy study of biological systems IEEE Trans. Dielectr. Electr. Insul. 10 728-53

[21] Kotnik T, Miklavcic D and Slivnik T 1998 Time course of transmembrane voltage induced by time-varying electric fields - a method for theoretical analysis and its application Bioelectrochem. Bioenerg. 45 3-16

[22] Foster K R 2000 Thermal and Nonthermal mechanisms of interaction of radio-frequency energy with biologica systems IEEE Trans. Plasma Sci. 28 15-23

[23] Jones T B 1995 Electromechanics of Particles (Cambridge: Cambridge University Press)

[24] Wang X-B, Pethig R and Jones T B 1992 Relationship of dielectrophoretic and electrorotational behaviour exhibited by polarized particles J. Phys. D: Appl. Phys. 25 905-12

[25] Wang X-B, Huang Y, Holzel R, Burt J P H and Pethig R 1993 Theoretical and experimental investigations of the interdependence of the dielectric, dielectrophoretic and electrorotational behaviour of colloidal particles J. Phys. D: Appl. Phys. 26 312-22

[26] Morgan H and Green N G 2003 AC Electrokinetics: Colloids and Nanoparticles (Herts, UK: Research Studies Press) 